DOE-287-CPP-25

\title{
Toward a Conformal Field Theory for the Quantum Hall Effect
}

\author{
Greg Nagao \\ Center for Particle Physics \\ The University of Texas \\ Austin, Texas 78712
}

\begin{abstract}
An effective Hamiltonian for the study of the quantum Hall effect is proposed. This Hamiltonian, which includes a "current-current" interaction has the form of a Hamiltonian for a conformal field theory in the large $N$ limit. An order parameter is constructed from which the Hamiltonian may be derived. This order parameter may be viewed as either a collective coordinate for a system of $N$ charged particles in a strong magnetic field; or as a field of spins associated with the cyclotron motion of these particles.
\end{abstract}




\section{Overview}

The IQHE is described by the Hamiltonian for a single particle in an external magnetic field. On the other hand, the FQHE is considered as a manifestation of a many-body effect of $N$ interacting particles in an external magnetic field [1]. The center of mass motion of these $N$ particles are usually neglected, assuming the fluctuations about the center of mass contain the key features neccessary to understand the fractional quantization of the Hall conductivity.

There have been many recent papers (e.g. [2, 30) relating vertex operators to the quantum Hall effect. In particular, it has been shown that the Laughlin wavefunction may be obtained as an $N^{\prime}$ - point correlation function of vertex operators. The conformal weights of these vertex operators are associated with fractional charges of the quasiparticle states. An attempt is made here to better understand these results in the context of a $2+1$ dimensional conformal field theory (CFT). Recalling that the conformal weights of vertex operators in string theory are associated with the center of mass (COM) motion of the string, we explicitly study the separation of the COM motion for a system of $N$ charged particles in a strong magnetic field. We find that the $N$-particle Hamiltonian (including the COM motion) may be diagonalized through the inclusion of a "current-current" interaction. It is then shown that this leads to an effective Hamiltonian resembling that of a CFT in the large $N$-limit. This suggests that we define a collective coordinate $\varphi_{L}(\zeta)$ in symmetric gauge. This collective coordinate may be viewed as a field of spins associated with the cyclotron motion of charged particles in the strong magnetic field. This "field of spins" may be taken as an order parameter for the study of the vacuum structure of the QHE.

\section{II. $N$ Non-interacting Particles in an External Magnetic Field}

The Hamiltonian usually taken to describe the QHE is that of $N$ noninteracting particles in an external magnetic field

$$
H_{0}=\frac{1}{2 \mu} \sum_{n=1}^{N} \vec{\pi}_{n}^{2}
$$

where

$$
\begin{aligned}
\vec{\pi}_{n} & \equiv \vec{p}_{n}+\frac{e}{c} \vec{A}\left(\vec{x}_{n}\right) \\
A^{i}\left(\vec{x}_{n}\right) & \equiv \frac{B}{2}\left(\epsilon^{i j} x_{n}^{j}+\frac{\partial \Lambda}{\partial x_{n}^{i}}\right)
\end{aligned}
$$

are the canonical momentum and vector potential in arbitrary gauge. Separating out the center of mass energy, the Hamiltonian becomes

$$
H_{0}=\frac{1}{2 \mu}\left(\vec{\pi}_{0}^{2}-\sum_{n \neq m=1}^{N} \vec{\pi}_{n} \cdot \vec{\pi}_{m}\right)
$$


where the center of mass $(\mathrm{COM})$ coordinates are

$$
\begin{gathered}
\vec{p}_{0} \equiv \sum_{n=1}^{N} \vec{p}_{n} \quad ; \quad \vec{x}_{0} \equiv \frac{1}{N} \sum_{n=1}^{N} \vec{x}_{n} \\
\vec{\pi}_{0} \equiv \vec{p}_{0}+\frac{e}{c} \vec{A}\left(N \vec{x}_{0}\right) \quad \Longrightarrow \quad \pi_{0}^{i}=p_{0}^{i}+\frac{\hbar N}{2 \ell^{2}}\left(\epsilon^{i j} x_{0}^{j}+\frac{1}{N^{2}} \frac{\partial \Lambda}{\partial x_{0}^{i}}\right) .
\end{gathered}
$$

Performing a canonical transformation to complex phase space coordinates, the Hamiltonian may be written as

$$
H_{0}=\hbar \omega\left(N \alpha_{0}^{*} \alpha_{0}-\sum_{n \neq m=1}^{N} \alpha_{n}^{*} \alpha_{m}\right)
$$

where $\omega \equiv \frac{e B}{\mu c}$ is the cyclotron frequency, $\ell^{2} \equiv \frac{e B}{\mu c}$ is the magnetic length and $\alpha$ is a dimensionless complex momentum

$$
\begin{aligned}
\alpha_{0} \equiv \frac{\ell}{\sqrt{2 N} \hbar}\left(\pi_{0}^{1}+i \pi_{0}^{2}\right) & ; & \alpha_{0}^{*} \equiv \frac{\ell}{\sqrt{2 N} \hbar}\left(\pi_{0}^{1}-i \pi_{0}^{2}\right) \\
\alpha_{n} \equiv \frac{\ell}{\sqrt{2} \hbar}\left(\pi_{n}^{1}+i \pi_{n}^{2}\right) & ; & \alpha_{n}^{*} \equiv \frac{\ell}{\sqrt{2} \hbar}\left(\pi_{n}^{1}-i \pi_{n}^{2}\right)
\end{aligned}
$$

with commutation relations (using $\left\{x_{0}^{i}, p_{0}^{j}\right\}=\delta^{i j}$ and $\left\{x_{n}^{i}, p_{n}^{j}\right\}=\delta^{i j}$ )

$$
\begin{aligned}
\left\{\pi_{0}^{i}, \pi_{0}^{j}\right\} & =\frac{N \hbar}{2 \ell^{2}}\left[2 \epsilon^{i j}+\frac{1}{N^{2}}\left(\left\{p_{0}^{i}, \frac{\partial \Lambda}{\partial x_{0}^{j}}\right\}+\left\{\frac{\partial \Lambda}{\partial x_{0}^{j}}, p_{0}^{j}\right\}\right)\right] \\
\left\{\pi_{n}^{i}, \pi_{n}^{j}\right\} & =\frac{\hbar}{2 \ell^{2}}\left[2 \epsilon^{i j}+\left\{p_{n}^{i}, \frac{\partial \Lambda}{\partial x_{n}^{j}}\right\}+\left\{\frac{\partial \Lambda}{\partial x_{n}^{j}}, p_{n}^{j}\right\}\right] \\
\left\{\alpha_{0}, \alpha_{0}^{*}\right\} & =\frac{-i}{2 \hbar}\left(2\left\{x_{0}^{1}, p_{0}^{1}\right\}+\frac{1}{N^{2}}\left\{\frac{\partial \Lambda}{\partial x_{0}^{1}}, p_{0}^{2}\right\}-\frac{1}{N^{2}}\left\{\frac{\partial \Lambda}{\partial x_{0}^{2}}, p_{0}^{1}\right\}\right) \\
\left\{\alpha_{n}, \alpha_{n}^{*}\right\} & =\frac{-i}{2 \hbar}\left(2\left\{x_{n}^{1}, p_{n}^{1}\right\}+\left\{\frac{\partial \Lambda}{\partial x_{n}^{1}}, p_{n}^{2}\right\}-\left\{\frac{\partial \Lambda}{\partial x_{n}^{2}}, p_{n}^{1}\right\}\right) .
\end{aligned}
$$

In symmetric gauge $(\Lambda=0)$ we thus have the expected commutator results $([x, p]=i \hbar)$ for annihilation-creation operators:

$$
\begin{array}{ccc}
\left\{\pi_{0}^{i}, \pi_{0}^{j}\right\}=\frac{N \hbar}{\ell^{2}} \epsilon^{i j} \quad ; \quad\left\{\pi_{n}^{i}, \pi_{n}^{j}\right\} \frac{\hbar}{\ell^{2}} \epsilon^{i j} \\
{\left[\pi_{0}^{i}, \pi_{0}^{j}\right]=i N\left(\frac{\hbar}{\ell}\right)^{2} \epsilon^{i j} \quad ;} & {\left[\pi_{n}^{i}, \pi_{n}^{j}\right]=i\left(\frac{\hbar}{\ell}\right)^{2} \epsilon^{i j}}
\end{array}
$$




$$
\begin{aligned}
\left\{\alpha_{0}, \alpha_{0}^{*}\right\}=\frac{-i}{\hbar} & ; & \left\{\alpha_{n}, \alpha_{n}^{*}\right\}=\frac{-i}{\hbar} \\
{\left[\alpha_{0}, \alpha_{0}^{\dagger}\right]=\frac{-i}{\hbar}\left[x_{0}^{1}, p_{0}^{1}\right]=1 } & ; & {\left[\alpha_{n}, \alpha_{n}^{\dagger}\right]=\frac{-i}{\hbar}\left[x_{n}^{1}, p_{n}^{1}\right]=1 . }
\end{aligned}
$$

\section{A Current-Current Interaction}

We now consider what happens if we include an interaction of the form

$$
\frac{1}{2 \mu} \sum_{n, m=1}^{N} \vec{\pi}_{n} \cdot \vec{\pi}_{m}
$$

will thus have the effect of diagonalizing the Hamiltonian

$$
\begin{aligned}
H & =H_{0}+\frac{1}{2 \mu} \sum_{n, m=1}^{N} \vec{\pi}_{n} \cdot \vec{\pi}_{m} \\
& =\frac{1}{2 \mu}\left[\vec{\pi}_{0}^{2}-\sum_{n \neq m=1}^{N} \vec{\pi}_{n} \cdot \vec{\pi}_{m}+\sum_{n, m=1}^{N} \vec{\pi}_{n} \cdot \vec{\pi}_{m}\right] \\
& =\frac{1}{2 \mu}\left(\vec{\pi}_{0}^{2}+\sum_{n=1}^{N} \vec{\pi}_{n}^{2}\right)
\end{aligned}
$$

This Hamiltonian can then be recognized as the generator of dilations $\left(L_{0}\right)$ for some Virasoro algebra (in the large $N$ limit) with $N$ playing the role of the inverse string tension $\left(\alpha^{\prime} \sim \frac{1}{T}\right)$. This can be most easily seen in terms of our complex momentum $\alpha$ :

$$
H=\hbar \omega\left(N \alpha_{0}^{*} \alpha_{0}+\sum_{n=1}^{N} \alpha_{n}^{*} \alpha_{n}\right) .
$$

In terms of an effective field theory (i.e. in the large $N$-limit), such a momentummomentum coupling (10) becomes a current-current interaction

$$
\vec{\pi} \cdot \vec{\pi} \longrightarrow \vec{j} \cdot \vec{j}
$$

As we shall see (41), such a current may be related to the conjugate momentum of a field associated with the collective coordinate for the $N$-particle system. We may thus identify this interaction as that which is neccessary to describe this system in terms of a CFT. The current here may be recognized as that of a Kac-Moody algebraf whose stress-energy tensor is given by the Sugawara construction. Since the only charges which are present in this model is that of the electric charge (which enters into the bosonic

\footnotetext{
${ }^{1}$ The involvement of a $U(1)$ Kac-Moody algebra in the QHE was first recognized by Wen [4] in a different manner.
} 
annihilation-creation operators). [f we expect this current to be the generator of a $U(1)$ Kac-Moody algebra.

\section{A Quantum Representation in Complex Phase Space}

Our effective Hamiltonian of the previous section contains the energy associated with COM momenta as well as that for fluctuations off the COM. Until now we have not found it neccessary to introduce configuration space variables. It has been enough to define commutation relations between conjugate momenta (or complex conjugate momenta). In order to give an explicit realization of this commutator algebra, however, it is convenient to introduce configuration space variables. In particular, since it is suggested that conformal symmetry plays an important role in this system, we find it convenient to define complex configuration space variables. Note configuration space variables enter directly into the Hamiltonian only through the vector potential. Defining a complex vector potential

$$
A \equiv A^{1}+i A^{2}
$$

with

$$
A^{i}=\frac{B}{2}\left(\epsilon^{i j} x^{j}+\frac{\partial \Lambda}{\partial x^{i}}\right) .
$$

allows us to evaluate the system in arbitrary gauge in terms of complex variables

$$
\begin{aligned}
\frac{e}{c} A & =\frac{\hbar}{\ell}\left(-i z+\frac{1}{2 \ell^{2}} \frac{\partial \Lambda}{\partial z^{*}}\right) \\
z & \equiv \frac{1}{2 \ell}\left(x^{1}+i x^{2}\right)
\end{aligned}
$$

This complex coordinate can thus be thought of as representing the position of a magnetic flux quantum. This can be seen more explicitly by noting that classically, the magnetic flux quantum is located at the bariocentric coordinate (or what we shall call a "psuedohole" associated with the charge conjugate operator [5]) around which the charge circulates. The bariocentric coordinate is given by

$$
\overrightarrow{\tilde{\pi}} \equiv \vec{p}-\frac{e}{c} \vec{A}(\vec{x})=\mathcal{C} \vec{\pi}
$$

where $\mathcal{C}$ is the charge conjugation operator; or in complex momentum coordinates

\footnotetext{
${ }^{2}$ The fermionic character of the electrons has been neglected in this quantization. It may be taken into account through statistical methods when calculating such physical quantities as the conductivity tensor.
} 


$$
\begin{aligned}
\beta & \equiv \frac{\ell}{\sqrt{2} \hbar}\left(\tilde{\pi}^{2}+i \tilde{\pi}^{1}\right) \\
& =i \alpha^{*}+\sqrt{2}\left(z^{*}-\frac{i}{2 \ell^{2}} \frac{\partial \Lambda}{\partial z}\right) .
\end{aligned}
$$

We may thus define coordinates for the COM and fluctuations off the COM coordinates

$$
\begin{aligned}
& z_{0} \equiv \frac{N}{2 \ell}\left(x_{0}^{1}+i x_{0}^{2}\right) \quad ; \quad z_{0}^{*}=\frac{N}{2 \ell}\left(x_{0}^{1}-i x_{0}^{2}\right) \\
& z_{n} \equiv \frac{1}{2 \ell}\left(x_{n}^{1}+i x_{n}^{2}\right) \quad ; \quad z_{n}^{*}=\frac{1}{2 \ell}\left(x_{n}^{1}-i x_{n}^{2}\right) \\
& \rho_{0} \equiv \frac{\ell}{N}\left(p_{0}^{1}-i p_{0}^{2}\right) \quad ; \quad \rho_{0}^{*}=\frac{\ell}{N}\left(p_{0}^{1}+i p_{0}^{2}\right) \\
& \rho_{n} \equiv \ell\left(p_{n}^{1}-i p_{n}^{2}\right) \quad ; \quad \rho_{n}^{*}=\ell\left(p_{n}^{1}+i p_{n}^{2}\right) \\
& p_{0}^{1}=\frac{N}{2 \ell}\left(\rho_{0}+\rho_{0}^{*}\right) \quad ; \quad p_{0}^{2}=\frac{i N}{2 \ell}\left(\rho_{0}-\rho_{0}^{*}\right)
\end{aligned}
$$

For the "quasiparticle" in symmetric gauge, we then have

$$
\begin{aligned}
& \pi_{0}^{1}=\frac{N}{2 \ell}\left[\left(\rho_{0}+\rho_{0}^{*}\right)+\frac{i \hbar}{N}\left(z_{0}^{*}-z_{0}\right)\right] \quad ; \quad \pi_{0}^{2}=\frac{i N}{2 \ell}\left[\left(\rho_{0}-\rho_{0}^{*}\right)+\frac{i \hbar}{N}\left(z_{0}^{*}+z_{0}\right)\right] \\
& \pi_{n}^{1}=\frac{1}{2 \ell}\left[\left(\rho_{n}+\rho_{n}^{*}\right)+i \hbar\left(z_{n}^{*}-z_{n}\right)\right] \quad ; \quad \pi_{n}^{2}=\frac{i}{2 \ell}\left[\left(\rho_{n}-\rho_{n}^{*}\right)+i \hbar\left(z_{n}^{*}+z_{n}\right)\right] \\
& \alpha_{0}=\sqrt{\frac{N}{2}} \frac{1}{\hbar}\left(\rho_{0}^{*}-\frac{i \hbar}{N} z_{0}\right) \quad ; \quad \alpha_{0}^{*}=\sqrt{\frac{N}{2}} \frac{1}{\hbar}\left(\rho_{0}+\frac{i \hbar}{N} z_{0}^{*}\right) \\
& \alpha_{n}=\frac{1}{\sqrt{2} \hbar}\left(\rho_{n}^{*}-i \hbar z_{n}\right) \quad ; \quad \alpha_{n}^{*}=\frac{1}{\sqrt{2} \hbar}\left(\rho_{n}+i \hbar z_{n}^{*}\right) ;
\end{aligned}
$$

and for the "quasipsuedohole"

$$
\begin{array}{rlrl}
\tilde{\pi}_{0}^{1}=\frac{N}{2 \ell}\left[\left(\rho_{0}+\rho_{0}^{*}\right)-\frac{i \hbar}{N}\left(z_{0}^{*}-z_{0}\right)\right] & ; & \tilde{\pi}_{0}^{2}=\frac{i N}{2 \ell}\left[\left(\rho_{0}-\rho_{0}^{*}\right)-\frac{i \hbar}{N}\left(z_{0}^{*}+z_{0}\right)\right] \\
\tilde{\pi}_{n}^{1}=\frac{1}{2 \ell}\left[\left(\rho_{n}+\rho_{n}^{*}\right)-i \hbar\left(z_{n}^{*}-z_{n}\right)\right] & ; & \tilde{\pi}_{n}^{2}=\frac{i}{2 \ell}\left[\left(\rho_{n}-\rho_{n}^{*}\right)-i \hbar\left(z_{n}^{*}+z_{n}\right)\right] \\
\beta_{0}=\sqrt{\frac{N}{2} \frac{1}{\hbar}\left(i \rho_{0}+\frac{\hbar}{N} z_{0}^{*}\right)} \quad ; & \beta_{0}^{*}=\sqrt{\frac{N}{2}} \frac{1}{\hbar}\left(-i \rho_{0}^{*}+\frac{\hbar}{N} z_{0}\right) \\
\beta_{n}=\frac{1}{\sqrt{2} \hbar}\left(i \rho_{n}+\hbar z_{n}^{*}\right) & ; & \beta_{n}^{*}=\frac{1}{\sqrt{2} \hbar}\left(-i \rho_{n}^{*}+\hbar z_{n}\right) .
\end{array}
$$


Differential operators may be defined

$$
\begin{aligned}
\frac{\partial}{\partial z_{0}} \equiv \frac{\ell}{N}\left(\frac{\partial}{\partial x_{0}^{1}}-i \frac{\partial}{\partial x_{0}^{2}}\right) & ; & \frac{\partial}{\partial z_{0}^{*}} & =\frac{\ell}{N}\left(\frac{\partial}{\partial x_{0}^{1}}+i \frac{\partial}{\partial x_{0}^{2}}\right) \\
\frac{\partial}{\partial z_{n}} \equiv \ell\left(\frac{\partial}{\partial x_{n}^{1}}-i \frac{\partial}{\partial x_{n}^{2}}\right) & ; & \frac{\partial}{\partial z_{n}^{*}} & =\ell\left(\frac{\partial}{\partial x_{n}^{1}}+i \frac{\partial}{\partial x_{n}^{2}}\right)
\end{aligned}
$$

giving rise to the usual quantum realizations

$$
\begin{aligned}
& \rho_{0}=-i \hbar \frac{\partial}{\partial z_{0}} \quad ; \quad \rho_{0}^{*}=-i \hbar \frac{\partial}{\partial z_{0}^{*}} \\
& \rho_{n}=-i \hbar \frac{\partial}{\partial z_{n}} \quad ; \quad \rho_{n}^{*}=-i \hbar \frac{\partial}{\partial z_{n}^{*}}
\end{aligned}
$$

and quantum commutators

$$
\begin{aligned}
{\left[z_{0}, \rho_{0}\right]=i \hbar } & ; & & {\left[z_{0}^{*}, \rho_{0}^{*}\right]=i \hbar } \\
{\left[z_{n}, \rho_{n}\right]=i \hbar } & ; & & {\left[z_{n}^{*}, \rho_{n}^{*}\right]=i \hbar }
\end{aligned}
$$

with all others vanishing. Our complex "momentum" operators become

$$
\begin{aligned}
& \alpha_{0}=-i \sqrt{\frac{N}{2}}\left(\frac{\partial}{\partial z_{0}^{*}}+\frac{1}{N} z_{0}\right) \quad ; \quad \alpha_{0}^{\dagger}=-i \sqrt{\frac{N}{2}}\left(\frac{\partial}{\partial z_{0}}-\frac{1}{N} z_{0}^{*}\right) \\
& \alpha_{n}=\frac{-i}{\sqrt{2}}\left(\frac{\partial}{\partial z_{n}^{*}}+z_{n}\right) \quad ; \quad \alpha_{n}^{\dagger}=\frac{-i}{\sqrt{2}}\left(\frac{\partial}{\partial z_{n}}-z_{n}^{*}\right) \\
& \beta_{0}=-\sqrt{\frac{N}{2}}\left(\frac{\partial}{\partial z_{0}}+\frac{1}{N} z_{0}^{*}\right) \quad ; \quad \beta_{0}^{\dagger}=-\sqrt{\frac{N}{2}}\left(\frac{\partial}{\partial z_{0}^{*}}-\frac{1}{N} z_{0}\right) \\
& \beta_{n}=\frac{1}{\sqrt{2}}\left(\frac{\partial}{\partial z_{n}}+z_{n}^{*}\right) \quad ; \quad \beta_{n}^{\dagger}=\frac{-1}{\sqrt{2}}\left(\frac{\partial}{\partial z_{n}^{*}}-z_{n}\right)
\end{aligned}
$$

leading to the normal ordered (as opposed to symmeterized) Hamiltonian

$$
H=\hbar \omega\left[N \alpha_{0}^{\dagger} \alpha_{0}+\sum_{n=1}^{N} \alpha_{n}^{\dagger} \alpha_{n}\right] .
$$

Energy eigenstates can then be obtained in the usual way. One finds the ground state wavefunction by requiring it be annihilated by all annihilation operators

$$
\begin{aligned}
& 0=\alpha_{0} \mid 0_{0}, 0_{1}, \ldots, 0_{N}> \\
& 0=\alpha_{0} \mid 0_{0}, 0_{1}, \ldots, 0_{N}>
\end{aligned}
$$


Labeling the degeneracy of states by "psuedoholes", the "highest weight" degenerate state can also be obtained by requiring it be annihilated by all "psuedohole" annihilation operators

$$
\begin{aligned}
& 0=\beta_{0} \mid 0_{0}, 0_{1}, \ldots, 0_{N} ; 0_{0}, 0_{1}, \ldots, 0_{N}> \\
& 0=\beta_{0} \mid 0_{0}, 0_{1}, \ldots, 0_{N} ; 0_{0}, 0_{1}, \ldots, 0_{N}>
\end{aligned}
$$

Appying creation operators then leads to the complex wavefunction

$$
\begin{aligned}
& \Psi_{\nu_{1}, \nu_{2}, \ldots, \nu_{N} ; \mu_{1}, \mu_{2}, \ldots, \mu_{N}}\left(z_{0}, z_{1}, \ldots, z_{N} ; z_{0}^{*}, z_{1}^{*}, \ldots, z_{N}^{*}\right) \\
& \quad \equiv\left\langle z_{0}, z_{1}, \ldots, z_{N} ; z_{0}^{*}, z_{1}^{*}, \ldots, z_{N}^{*} \mid \nu_{1}, \nu_{2}, \ldots, \nu_{N} ; \mu_{1}, \mu_{2}, \ldots, \mu_{N}\right\rangle \\
& \quad=f_{\nu_{1}, \nu_{2}, \ldots, \nu_{N} ; \mu_{1}, \mu_{2}, \ldots, \mu_{N}}\left(z_{0}, z_{1}, \ldots, z_{N} ; z_{0}^{*}, z_{1}^{*}, \ldots, z_{N}^{*}\right) e^{-\frac{\left|z_{0}\right|^{2}}{N}-\sum_{n=1}^{N}\left|z_{n}\right|^{2}}
\end{aligned}
$$

where $\nu_{n}$ and $\mu_{n}$ label the energy levels and "psuedoholes", respectively

$$
\begin{array}{lll}
\alpha_{0}^{\dagger} \alpha_{0}\left|\nu_{0}>=\nu_{0}\right| \nu_{0}>\quad & ; & \alpha_{n}^{\dagger} \alpha_{n}\left|\nu_{n}>=\nu_{n}\right| \nu_{n}> \\
\beta_{0}^{\dagger} \beta_{0}\left|\mu_{0}>=\mu_{0}\right| \mu_{0}>\quad & ; & \beta_{n}^{\dagger} \beta_{n}\left|\mu_{n}>=\mu_{n}\right| \mu_{n}>
\end{array}
$$

The functional form of the pre-exponential factor $f$ at this point in undetermined; however it should obviously exhibit the proper statistics of the $N$ particles. Such statistical phase factors might be incorporated through a modification of the oscillator commutation relations (as in the generalization of the bosonic string to the fermionic and superstring); however we content ourselves here with the bosonic formalism in which the bosonic vertex operators allow for anyonic statistics.

\section{Commutation Relations and the OPE}

In the procedure for first quantization, the usual methods of functions and derivatives were used as a realization of the commutator algebra for quantum operators. In the field-theoretic formulation of the many-body system, it becomes convenient to realize the quantum algebra through a mode expansion. In the language of CFT the mode expansion is know as the operator product expansion (OPE). Another technique of CFT which we shall find useful to implement is that of the construction of primary fields or vertex operators. In particular, winding state vertex operators have the important property that they create coherent states for the COM motion of the "quasiparticle".

To motivate the field-theoretic formulation, we define a quasiparticle field $\pi(\zeta)$ in terms of an $N$-particle mode expansion

$$
\pi(\zeta) \sim \sum_{n=0}^{N} \alpha_{n} \zeta^{n-1}
$$


where $\left\{\alpha_{n}\right\}_{n=0}^{N}$ are some $N+1$ independent modes of the quasiparticle which may be taken to be the creation-annihilation operators of the COM conjugate momenta $\left(\alpha_{0} \sim\right.$ $\left.\pi_{0}^{1}+i \pi_{0}^{2}\right)$ and the conjugate momenta for each constituent particle of the quasiparticle $\left(\alpha_{n} \sim \pi_{n}^{1}+i \pi_{n}^{2}\right)$. Here $\zeta$ is a complex coordinate. In a holomorphic representation where Fourier modes are orthogonal, the coefficient $\zeta^{n}$ may be loosely interpreted as the relative coordinate of the $n$th particle from the COM of the quasiparticle. It is related to the fluctuation of the magnetic field $(\vec{p} \cdot \vec{A})$ from the average magnetic field strength around the COM. To arrive at this interpretation, we consider our effective Hamiltonian to be

$$
\begin{aligned}
H & =\frac{1}{2 \mu}\left(\vec{\pi}_{0}^{2}+\sum_{n=1}^{N} \vec{\pi}_{n}^{2}\right) \\
& =\hbar \omega\left(N \alpha_{0}^{*} \alpha_{0}+\sum_{n=1}^{N} \alpha_{n}^{\prime *} \alpha_{n}^{\prime}\right)
\end{aligned}
$$

where

$$
\begin{gathered}
\vec{\pi}_{n}^{\prime}=\vec{\pi}_{n}-\vec{\pi}_{0} \quad ; \quad \vec{\pi}_{n}^{\prime}=\vec{p}_{n}^{\prime}+\frac{e}{c} \vec{A}^{\prime}\left(\vec{x}_{n}\right) \\
\vec{p}_{n}^{\prime}=\vec{p}_{n}-\vec{p}_{0} \quad ; \quad \vec{A}^{\prime}\left(\vec{x}_{n}\right) \equiv \vec{A}\left(\vec{x}_{n}\right)-\vec{A}\left(\vec{x}_{0}\right) \\
\alpha_{n}^{\prime} \equiv \frac{1}{\sqrt{2} \hbar}\left(\rho_{n}^{* *}+\frac{e \ell}{c} A^{\prime}\left(z_{n}\right)\right)=\frac{1}{\sqrt{2}}\left(\frac{\rho_{n}^{\prime *}}{\hbar}+\frac{2 \pi \ell}{\Phi_{0}} A^{\prime}\left(z_{n}\right)\right) \\
\alpha_{n}^{\prime *} \equiv \frac{1}{\sqrt{2} \hbar}\left(\rho_{n}^{\prime}+\frac{e \ell}{c} A^{\prime *}\left(z_{n}\right)\right)=\frac{1}{\sqrt{2}}\left(\frac{\rho_{n}^{\prime}}{\hbar}+\frac{2 \pi \ell}{\Phi_{0}} A^{\prime *}\left(z_{n}\right)\right) .
\end{gathered}
$$

From the correspondence principle, we then quantize by defining

$$
\rho_{n}^{\prime} \equiv-i \hbar \frac{\partial}{\partial z_{n}^{\prime}} \quad ; \quad \rho_{n}^{*} \equiv-i \hbar \frac{\partial}{\partial z_{n}^{\prime *}}
$$

We may alternatively represent these quantum operators using the techniques of complex variables and the residue theorem for derivatives

$$
\begin{aligned}
2 \pi i f\left(\zeta_{0}\right) & =\oint \frac{f(z)}{\zeta-\zeta_{0}} d \zeta \\
2 \pi i f^{(n)}\left(\zeta_{0}\right) & =\oint \frac{f(z)}{\left(\zeta-\zeta_{0}\right)^{n+1}} d \zeta .
\end{aligned}
$$

\footnotetext{
${ }^{3}$ This quantization is analagous to that of the background field method of QFT. Here the dynamical degrees of freedom are those of the fluctuations of the background COM.
} 
Thus, if we represent

$$
\rho_{n}^{\prime} \sim \frac{1}{\zeta^{n+1}} \quad ; \quad z_{n}^{\prime} \sim \zeta^{n}
$$

then the canonical commutation relations can be represented by a contour integral

$$
\begin{aligned}
{\left[z_{n}^{\prime}, \rho_{m}^{\prime}\right] } & \equiv \frac{1}{2 \pi i} \oint \zeta^{n} \rho_{m}^{\prime}(\zeta) d \zeta \\
& \sim \frac{1}{2 \pi i} \oint \zeta^{n} \zeta^{-m-1} d \zeta \sim \delta_{n m}
\end{aligned}
$$

\section{Collective Coordinates for Quasiparticle States}

In the previous section we showed how the $N$-particle Hamiltonian could be expressed nicely in terms of a set of COM oscillators and fluctuations from the COM. From this point of view the above Hamiltonian is an $N$-particle Hamiltonian; on the other hand, we would like to reinterpret this Hamiltonian as that of a single quasiparticle. For this purpose it is convenient to define a set of collective coordinates for excitations of the quasiparticle (as opposed to the individual excitations of the individual particles).

Assuming the current-current interaction gives rise to coherent $N$-particle states, we define collective coordinates

$$
\begin{aligned}
\varphi_{L}(\zeta) & \equiv \varphi_{0_{L}}+i \sqrt{N} \alpha_{0} \ln \zeta+\sum_{n=1}^{N} \frac{1}{n}\left(\alpha_{n}(\tau) \zeta^{n}+\alpha_{n}^{*}(\tau) \zeta^{-n}\right) \\
\pi(\zeta) & \equiv \frac{\partial \varphi(\zeta)}{\partial \zeta}=i \sqrt{N} \frac{\alpha_{0}}{\zeta}+\sum_{n=1}^{N} \frac{1}{\zeta}\left(\alpha_{n} \zeta^{n}-\alpha_{n}^{*} \zeta^{-n}\right) .
\end{aligned}
$$

These collective coordinates may alternatively be viewed as a field of "spins" associated with the cyclotron motion of the charged particles in the strong external magnetic field. From this point of view, the annihilation - creation operators $\left(\alpha_{n}, \alpha_{n}^{\dagger}\right)$ may be viewed as spin operators annihilating and creating spins whose spin varies with $n$.

It is useful to define creation operators with negative index

$$
\alpha_{n}^{*} \equiv \alpha_{-n}
$$

allowing us to write our mode expansion in more compact form

$$
\begin{aligned}
\varphi_{L}(\zeta) & \equiv \varphi_{0_{L}}+i \sqrt{N} \alpha_{0} \ln \zeta+\sum_{n=-N}^{\prime} \frac{1}{n} \alpha_{n} \zeta^{n} \\
\pi_{L}(\zeta) & \equiv \frac{\varphi_{L}}{\partial \zeta}=\frac{1}{\zeta}\left[i \sqrt{N} \alpha_{0}+\sum_{n=-N}^{N} \alpha_{n} \zeta^{n}\right]
\end{aligned}
$$


where $\sum^{\prime}$ indicates a sum over non-zero modes.

In our attempt to understand the critical behaviour of this system of charged particles in a strong magnetic field, it is natural to define a stress-energy tensor from this collective coordinate $\varphi_{L}(\zeta)$ which serves as our order parameter

$$
T_{\zeta \zeta} \equiv \pi_{L}^{2}=\frac{\varphi_{L}}{\partial \zeta} \frac{\varphi_{L}}{\partial \zeta}
$$

We may then associate a Hamiltonian density for an effective field theory with this stressenergy tensor. A natural way to do this is suggested by the methods of CFT. Assuming that the ground state of our system exhibits an aproximate conformal invariance associated with a critical value for the magnetic field strength $B_{c}$, it is then appropriate to identify our Hamiltonian density with the second order moment of the stress-energy tensor. f This second order moment may be identified with the generator of dilations $L_{0}$ in a CFT. The $n$-th order moments are called Virasoro generators. They are the generators of $(n+2)$-th order conformal transformations in such a field theory [6] defined by

$$
T(\zeta) \equiv \sum_{n=-\infty}^{\infty} \frac{L_{n}}{\zeta^{n+2}}
$$

Expanding our stress-energy tensor, we then have

$$
\begin{aligned}
T(\zeta)= & \frac{1}{\zeta^{2}}\left[i \sqrt{N} \alpha_{0}+\sum_{n=1}^{N}\left(\alpha_{0} \zeta^{n}-\alpha^{*} \zeta^{-n}\right)\right]^{2} \\
= & \frac{1}{\zeta^{2}}\left\{-N \alpha_{0}^{2}+2 i \sqrt{N} \alpha_{0} \sum_{n=1}^{N}\left(\alpha_{n} \zeta^{n}-\alpha_{n}^{*} \zeta^{-n}\right)\right. \\
& \left.+\sum_{n, m=1}^{N}\left[\alpha_{n} \alpha_{m} \zeta^{n+m}+\alpha_{n}^{*} \alpha_{m}^{*} \zeta^{-n-m}-\left(\alpha_{n}^{*} \alpha_{m} \zeta^{m-n}+\alpha_{n} \alpha_{m}^{*} \zeta^{n-m}\right)\right]\right\}
\end{aligned}
$$

Thus, in the large $N$-limit, we have

$$
L_{0} \approx-N \alpha_{0}^{2}-\sum_{n=1}^{N}\left[\left(\alpha_{n} \alpha_{-n}+\alpha_{n}^{*} \alpha_{-n}^{*}\right)+\left(\alpha_{n}^{*} \alpha_{n}+\alpha_{n} \alpha_{n}^{*}\right)\right]
$$

which, when "normal ordered" in operator form reduces to

$$
L_{0} \approx N \alpha_{0}^{*} \alpha_{0}+\sum_{n=-N}^{N} \alpha_{n}^{*} \alpha_{n} .
$$

We thus define our Hamiltonian (density) as

\footnotetext{
${ }^{4}$ In this way, an integration over the two-dimensional configuration space will yield the energy of the system.
} 


$$
\begin{aligned}
H & =N \alpha_{0}^{*} \alpha_{0}+\eta \\
\eta & \equiv \sum_{n=-N}^{\prime} \alpha_{n}^{*} \alpha_{n} \\
H \mid \text { phys }> & =\left(N \alpha_{0}^{*} \alpha_{0}+\eta\right) \mid \text { phys }>
\end{aligned}
$$

where energy eigenstate may be labeled by the COM momentum $\alpha_{0}$ and $\mid$ phys $>$ are "physical" states whose characteristics and significance are yet to be determined. In particular we expect BRST transformations to play an important role here. BRST transformations $(Q)$ are related to the reparameterization invariance associated with the Virasoro algebra. In this formalism, physical states are related to "exact" states whose $Q^{2}$ eigenvalue vanishes. Physical "null" states are related to closed, but not exact physical states. We expect these null states to be related to states which are just "gauge equivalent" to the exact states. Assuming the techniques of CFT to be applicable, we thus expect the approximate conformal symmetry to tell us something about the transformation (and thus internal symmetry) properties of the wavefunctions associated with the various excitations of the system.

\section{Collective Coordinates for "Quasipsuedoholes"}

We find it useful to define collective coordinates for "quasipsuedoholes" in order to understand the symmetries associated with the Landau degeneracy. Recall that for a single (two-dimensional) particle in an external magnetic field, there exists a conserved quantity $(\tilde{\pi})$ associated with the bariocentric coordinate for the cyclotronic motion of the particle. Since this conserved quantity can be represented by the charge conjugation of the canonical momentum, we have called this a "psuedohole".

Following the quasiparticle case, we define

$$
\begin{aligned}
\tilde{\pi}\left(\zeta^{*}\right) & \equiv \frac{\partial \varphi_{R}\left(\zeta^{*}\right)}{\partial \zeta^{*}} \\
\varphi_{R}\left(\zeta^{*}\right) & \equiv \varphi_{0_{R}}+i \sqrt{N} \beta_{0} \ln \zeta^{*}+\sum_{n=-N}^{N} \frac{1}{n} \beta_{n} \zeta^{* n} \\
\tilde{T}_{\zeta^{*} \zeta^{*}} & =\tilde{\pi}^{2} \\
\tilde{H} & =\tilde{N} \beta_{0}^{*} \beta_{0}+\sum_{n=-N}^{N} \beta_{n}^{*} \beta_{n} \sim \tilde{L}_{0}
\end{aligned}
$$

where $\tilde{L}_{0}$ is the second order moment of the stress energy tensor $\tilde{T}_{\zeta^{*} \zeta^{*}}$. This quasipsuedohole Hamiltonian may be considered to describe the "dynamics" of the bariocentric

\footnotetext{
${ }^{5}$ The conserved charges have different interpretations in different gauges. Our description in terms of a "psuedohole" comes from the symmetric gauge choice. This obviously generalizes to other gauges.
} 
coordinates associated with the position of a magnetic flux quantum. Since the complex coordinates label the positions of the particles (as in the usual formulations of CFT's), the generator of rotations is easily shown to be

$$
L_{3} \equiv \hbar\left(L_{0}-\tilde{L}_{0}\right)
$$

\section{Conclusion}

We have shown how the addition of a "current-current" interaction between particles leads to a diagonalization of the $N$-particle Hamiltonian. Interpreting this interaction term as that associated with fluctuations from the COM leads to a field theoretical Hamiltonian resembling that of a $2+1$ dimensional CFT in the large $N$ limit. A similar field theoretical Hamiltonian may also be constructed for the states associated with the "psuedohole" operators. It should thus be possible to construct a $2+1$ dimensional field theory incorporating the dynamics of both particle and "psuedohole" fluctuations. Such a field theory could be useful in developing a nonlinear framework from which it might be possible to understand the connections between different ground state vacuua associated with the different phases of the QHE.

\section{Acknowledgements}

It is a pleasure to thank S. Thomas, J. Gaite and Q. Niu for helpful discussions. This work was supported by DOE grant DOE-287-CPP-25. 


\section{References}

1. Laughlin R.B., Phys. Lett. 50 (1983) 1395.

2. Fubini S., Vertex Operators and the Quantum Hall Effect, CERN-TH. 5922/90. Fubini S. and Lütken C.A., Vertex Operators in the Fractional Quantum Hall Effect, CERN-TH. 5960/90.

3. Stone M., Vertex Operators in the Quantum Hall Effect, IL- TH-90-\# 32.

Balatsky A. and Stone M., Vertex Operators and Spinon Edge Excitations in the Spin-Singlet Quantum Hall Effect, IL-TH-90-\#35.

Dunne G.V., Lerda A. and Trugenberger C.A., Landau Levels and Vertex Operators for Anyons, CTP \# 1938 (and LANL 91-535).

4. Wen X.G., Gapless Boundary Excitations in the Quantum Hall States and in the Chiral Spin States, NSF-ITP-89-157 (unpublished).

5. Nagao G., "Psuedoholes", Gauge Constraints and Critical Phenomena in the Quantum Hall Effect, unpublished.

6. Belavin A.A., Polyakov A.M. and Zamolodchikov A.B., Infinite Conformal Symmetry In Two-Dimensional Quantum Field Theory, Nucl. Phys. B 241 (1984) 333. 